\title{
Wigner Crystallization in Rapidly Rotating 2D Dipolar Fermi Gases
}

\author{
M.A. Baranov \\ Van der Waals-Zeeman Instituut, Unversiteit van Amsterdam, \\ Valckenierstraat 65, 1018 XE Amsterdam, The Netherlands and \\ C Kurchatov Institute, Kurchatov sq. 1. 115582 Moscow, Russian Federation \\ H. Fehrmann \\ Institut für Theoretische Physik, Leibniz Universität Hannover, Appelstraße 2, 30167 Hannover, Germany \\ M. Lewenstein \\ ICREA and ICFO-Institut de Ciències Fotòniques, Castelldefels 08860, Spain
}

\begin{abstract}
We study the competition between the Wigner crystal and the Laughlin liquid states in an ultracold quasi two-dimensional rapidly rotating polarized fermionic dipolar gas, and find that the Wigner crystal has a lower energy below a critical filling factor. We examine the quantum crystal to liquid transition for different confinements in the third direction. Our analysis of the phonon spectra of the Wigner crystal with the account of phonon-phonon interactions also shows the stability of the Wigner crystal for sufficiently low filling factors $(\nu<1 / 7)$.
\end{abstract}

Recently a remarkable progress has been made in experimental studies of strongly correlated systems of ultra cold gases. A seminal breakthrough was the observation of Mott insulator-superfluid transitions in Bose gases in optical lattices [1], followed by studies of low dimensional gases, fermionic superfluidity or ultracold disordered gases (for recent reviews of this very rapidly developing area see [2, 3]). A particularly fascinating route toward creation of strongly correlated states is the one that uses rapidly rotating gases. The rotation is formally equivalent to a magnetic field (in the rotational frame), which reorganizes free-particle states into discrete highly degenerate Landau levels. As a result, the properties of the system become very sensitive to interparticle interactions, in complete analogy to the Fractional Quantum Hall Effect (FQHE) for electrons [4], that exhibits a variety of strongly correlated states, among which the Laughlin liquid [5], and the Wigner crystal [6] are probably the most famous. This analogy has been pointed out in the context of ultracold atomic gases with short range interactions in Refs. [7], but an experimental observation of the FQHE in this case is difficult, partially due to smallness of the energy gap separating a Laughlin state from the so called quasi-hole excitations. It was recently argued [8] that these problems may be overcome in rotating quasi-2D polarized dipolar gases, in which the interparticle interaction has a long-range part that puts dipolar systems somewhat in between atomic systems with short-range interactions and electron systems with Coulomb interactions. As a result, the Laughlin state for the filling factor $\nu=1 / 3[32]$ was predicted to be incompressible with gapped excitations [8].

Several other unexpected predictions have been already made for rotating dipolar bosonic gases. The rotating dipolar condensates in the mean-field regime exhibit novel forms of vortex lattices: square, "stripe crys- tal", and "bubble crystal" lattices [9, 10]. On the other hand, Rezayi et al. [11] have recently shown that a small amount of dipole-dipole interactions stabilizes the so called bosonic Rezayi-Read strongly correlated state at $\nu=3 / 2$ whose excitations are both fractional, and non-Abelian.

The dipolar interactions could also lead to a crystal ground state in a quasi 2D system similar to the electron Wigner crystal. In the case of an electron gas, both in the presence and in the absence of a magnetic field, Wigner crystal becomes energetically favorable and stable for low filling factors: this is due to the fact that at low densities Coulomb interactions $(\sim 1 / R$, where $R$ is a typical length scale) win over the kinetic energy $\left(\sim 1 / R^{2}\right)$ (cf. [12]). For non-rotating dipolar gases (where interactions scale as $\left.1 / R^{3}\right)$, it was argued [8] that the ground state has a crystal order at high densities, as shown in Refs. [13]. Remarkably, in the case of a rotating dipolar gas, the Wigner crystal phase is expected at low densities [8]. Therefore, the dipolar gases, being to some extend similar to electrons, present nevertheless a novel class of physical systems exhibiting quantum liquid to crystal transition. This opens an unprecedented possibility for the observation and detailed studies of this transition in clean and well-controlled conditions. It is especially appealing in view of the recent experimental realization of the dipolar Bose condensate of Chromium [14, 15], and progress in trapping and cooling of dipolar molecules 16$]$.

In this paper we investigate the existence of a Wigner crystal phase in a rapidly rotating gas of polarized dipolar fermions by comparing it with the competing liquid states. We then examine the stability of the Wigner crystal by incorporating phonon-phonon interactions, and identify the border of stability with the appearance of purely imaginary phonon frequencies. It appears that the Wigner crystal is the stable ground state of the sys- 
tems for low filling factors, typically for $\nu<1 / 7$.

We consider a gas of fermionic polarized dipolar particles in a rotating cylindrical trap, tightly confined and polarized along the axis of rotation. For a sufficiently strong confinement in the axial direction, all particles are in the ground state of the axial motion and we can write the many-body wave function in the form

$$
\psi_{3 \mathrm{D}}\left(\left\{\mathbf{r}_{i}, \xi_{i}\right\}\right)=\psi_{2 \mathrm{D}}\left(\left\{\mathbf{r}_{i}\right\}\right)(l \sqrt{\pi})^{-N / 2} \exp \left(-\sum_{i} \xi_{i}^{2} / 2 l^{2}\right),
$$

where $\mathbf{r}_{i}=(x, y)$ and $\xi_{i}$ are radial and axial coordinates of the particles, respectively, $l$ is the extension in the axial direction, $N$ is the total number of particles, and $\psi_{2 D}$ is the wave function of the system in the $x y$-plane. The confinement in the axial direction also modifies the interparticle interaction in the $2 D$-plane. For two dipoles separated by a distance $r$ in the $2 D$-plane, the effective interaction potential is

$$
v_{2 \mathrm{D}}(r)=\frac{d^{2}}{l^{3} \sqrt{2 \pi}} \int_{0}^{\infty} d \xi \sqrt{\frac{\xi}{(\xi+1)^{3}}} \exp \left(-\xi \frac{r^{2}}{2 l^{2}}\right) \quad .
$$

For $r \gg l$ the potential is $v_{2 \mathrm{D}} \approx d^{2} / r^{3}$, while for $r<l$ the effective potential increases logarithmically, $v_{2 \mathrm{D}} \approx$ $\left(d^{2} / l^{3}\right) \sqrt{2 / \pi} \ln (l / r)$. The wave function $\psi_{2 D}$ obeys the Schrödinger equation with the effective 2D Hamiltonian that in the rotational frame reads

$$
H=-\sum_{i}\left(\frac{\hbar^{2}}{2 m} \Delta_{i}+\frac{m}{2} \omega_{\perp}^{2} \mathbf{r}_{i}^{2}\right)-\Omega L_{z}+V_{D}
$$

where $\Omega$ is the rotational frequency, $\omega_{\perp}$ is the radial trap frequency, $V_{D}=\sum_{i<j} v_{2 D}\left(\left|\mathbf{r}_{i}-\mathbf{r}_{j}\right|\right)$ describes the dipole-dipole interparticle interaction, and $L_{z}$ is the $z$ component of the total angular momentum. The Hamiltonian (11) can be rewritten in the form

$$
H=\sum_{i} \frac{1}{2 m}\left(-i \hbar \nabla_{i}-\mathbf{A}_{i}\right)^{2}-\left(\Omega-\omega_{\perp}\right) L_{z}+V_{\mathrm{D}}
$$

with $\mathbf{A}_{i}=m \omega_{\perp} \mathbf{e}_{z} \times \mathbf{r}_{i}$ and, therefore, it formally describes a system of charged particles in a constant magnetic field with the cyclotron frequency $\omega_{c}=2 \omega_{\perp}$. For non-interacting particles $\left(V_{\mathrm{D}}=0\right)$ in the regime of the critical rotation $\left(\omega_{\perp}=\Omega\right)$, the properties of the Hamiltonian (2) are well known; it's spectrum consists of highly degenerate levels $E_{n}=\hbar \omega_{c}(n+1 / 2)$ called Landau levels. In the following, we restrict ourself to a system of particles occupying only the lowest Landau level.

The wave function of a non-correlated Wigner crystal on the lowest Landau level is [17]:

$$
\Psi_{\mathrm{C}}\left(\left\{\mathrm{z}_{i}\right\}\right) \sim \mathcal{A} \prod_{i} \exp \left[\frac{-1}{4 l_{0}^{2}}\left(\left|z_{i}-R_{i}\right|^{2}+z_{i} R_{i}^{*}-z_{i}^{*} R_{i}\right)\right],
$$

where $z_{i}=x_{i}+i y_{i}$ is the complex representation of the $2 D$ vector $\mathbf{r}_{i}, R_{i}$ the (complex) lattice site, $l_{0}=\sqrt{\hbar / m \omega_{c}}$ the magnetic length, and $\mathcal{A}$ the antisymmetrization operator, which can be omitted for sufficiently low filling factors $\nu=2 \pi l_{0}^{2} n$. One can check that similar to the case of classical dipoles, the energy is minimal for a triangular lattice with particles centered at positions $\mathbf{R}_{i}=l_{1} \mathbf{b}_{1}+l_{2} \mathbf{b}_{2}$, where $l_{1,2}$ are integers, $\mathbf{b}_{1}=a(0,1)$, $\mathbf{b}_{2}=a(\sqrt{3}, 1) / 2$, and $a$ is the lattice constant determined by the density $n$ of the gas, $a^{2}=2 / \sqrt{3} n=$ $4 \pi l_{0}^{2} / \sqrt{3} \nu$. The energy of the crystal state equals

$$
\begin{aligned}
U_{\mathrm{C}} & =\left\langle\Psi_{\mathrm{C}}\left|V_{D}\right| \Psi_{\mathrm{C}}\right\rangle \approx d^{2} n^{3 / 2}(0.2823+0.2146 \beta \\
& \left.+0.3388 \beta^{2}+0.7456 \beta^{3}+2.0676 \beta^{4}+\ldots\right),
\end{aligned}
$$

where $\beta=\pi n\left(2 l_{0}^{2}-l^{2}\right)$ and the first term corresponds to the energy of classical point-like dipoles, $E_{\mathrm{cl}}=$ $\sum_{i<j} d^{2} /\left|l_{1} \mathbf{b}_{1}+l_{2} \mathbf{b}_{2}\right|^{3}=5.513 d^{2} / a^{3}$.

The energy of the Laughlin state (for a small number of particles interacting via dipolar forces, recent computations show a remarkable overlap of the exact ground state with the Laughlin state [18]) characterized by an odd integer $M=1 / \nu$ is

$$
U_{\mathrm{L}}=\frac{\nu}{2} \int_{0}^{\infty} r g(r) v_{2 \mathrm{D}}(r) d r,
$$

where $g(r)$ is the pair correlation function. We calculated this functions and the energies for odd $M$ from 1 to 19 for a gas of 512 particle using a Monte Carlo method.

We now compare the energies of the Wigner crystal and of the Laughlin liquid for different filling factors $\nu$ and extensions $l$ in the axial direction. The results are presented in Fig. 1. They show that below some critical value $\nu_{c}$, which depends on $l$, the Wigner crystal has a lower energy than the Laughlin liquid and, therefore, for $\nu<\nu_{c}$ the ground state is expected to be a crystal. Note that in obtaining the critical filling factor we compare the energies of two simplest trail wave functions for liquid and crystal states. A better estimate could follow from considering the wave functions of quantum Hall liquids of composite fermions [19] with the filling factors closer to the critical one than primary Laughins $1 / M$, and of the correlated Wigner crystals [20]. We, however, do not expect a significant change of our result because for a dilute system the two considered wave functions already catch the most important features of the interparticle interaction.

Let us now approach the liquid-crystal transition starting from the crystal phase. For this purpose we consider the phonon spectrum with taking into account phononphonon interactions (anharmonicity effects). The appearance of purely imaginary phonon frequencies will indicate the instability of the crystal and, therefore, the transition to a liquid state.

In the harmonic approximation the phonon eigenfrequencies can be obtained from the dynamic equations for displacements $u_{\alpha \mathbf{l}}$ of particles along the $\alpha$-axis from 


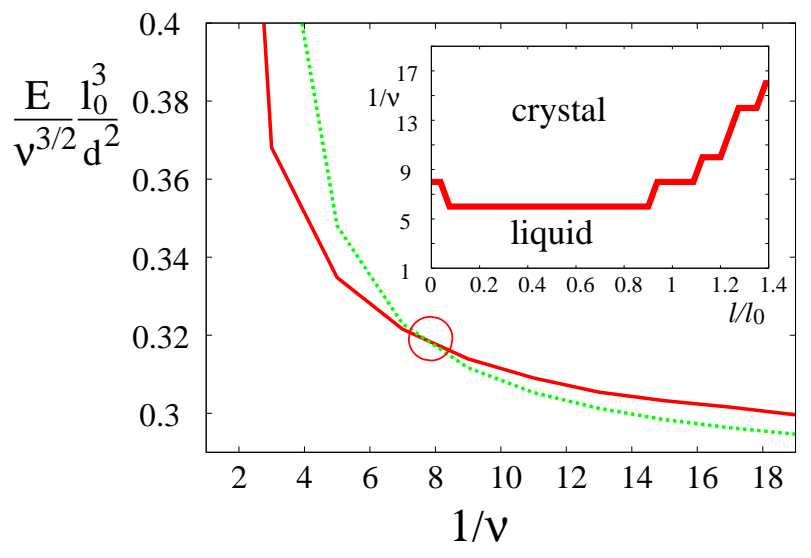

Figure 1: Energy per particle for the Wigner crystal (dotted line) and for the Laughlin state (solid line) as a function of the filling factor for $l=0$. The insert shows the critical filling factor as a function of the extension in the $z$-direction.

their equilibrium positions $\mathbf{R}_{\mathbf{1}}$ in the lattice 21]

$$
m \ddot{u}_{\alpha \mathbf{l}}=\sum_{\beta \mathbf{l}^{\prime}} \Phi_{\alpha \mathbf{l}, \beta \mathbf{l}^{\prime}}^{(2)} u_{\beta \mathbf{l}^{\prime}}+\omega_{c} \varepsilon_{\alpha \beta} \dot{u}_{\beta \mathbf{l}^{\prime}}
$$

where $\Phi_{\alpha \mathbf{l}, \beta \mathbf{1}^{\prime}}^{(2)}=\partial^{2} U_{\mathrm{C}} / \partial R_{\alpha \mathbf{l}} \partial R_{\beta \mathbf{1}^{\prime}}$ is the dynamical matrix, $\alpha, \beta=x, y$ and $\varepsilon_{\alpha \beta}$ is the antisymmetric tensor, $\varepsilon_{x y}=1$. The last term in Eq. (3) corresponds to the Coriolis (Lorentz) force due to the rotation (magnetic field).

Without rotation, $\omega_{c}=0$, Eq. 3 in the quasimomentum representation reads:

$$
\omega^{2} \tilde{u}_{\alpha}(\mathbf{k})=\sum_{\beta} F_{\alpha \beta}(\mathbf{k}) \tilde{u}_{\beta}(\mathbf{k})
$$

where $m F_{\alpha \alpha^{\prime}}(\mathbf{k})=\sum_{\mathbf{l}} \exp \left[-i \mathbf{k}\left(\mathbf{R}_{\mathbf{l}}-\mathbf{R}_{\mathbf{l}^{\prime}}\right)\right] \Phi_{\alpha \mathbf{l}, \beta \mathbf{l}^{\prime}}^{(2)}$ is the Fourier transform of $\Phi_{\alpha \mathbf{l}, \beta \mathbf{l}^{\prime}}^{(2)}$, and the eigenvalues $\omega_{s}^{2}(\mathbf{k})$, $s=T, L$ of $F_{\alpha \alpha^{\prime}}(\mathbf{k})$ determines the frequencies of the transversal $(T)$ and longitudinal $(L)$ phonons. The phonon frequencies for $l=0$ are shown in Fig. 2, They are linear in $k$ for $k \ll a^{-1}: \omega_{T} \approx(3 / \sqrt{8}) \omega_{d} a k$ and $\omega_{L} \approx \sqrt{11} \omega_{T}$, where $\omega_{d}=\sqrt{d^{2} / m a^{5}}$ sets the typical value for the frequency of phonons.

For a rotating crystal, $\omega_{c} \neq 0$, the phonon spectrum becomes 21, 22]

$$
\omega_{ \pm}^{2}=\frac{\omega_{L}^{2}+\omega_{T}^{2}+\omega_{c}^{2}}{2} \pm \frac{1}{2} \sqrt{\left(\omega_{L}^{2}+\omega_{T}^{2}+\omega_{c}^{2}\right)^{2}-4 \omega_{L}^{2} \omega_{T}^{2}} .
$$

In the case $\omega_{c} \gg \omega_{s}$, one has $\omega_{+} \approx \omega_{c}$ and $\omega_{-} \approx$ $\omega_{L} \omega_{T} / \omega_{c}$.

Higher orders (anharmonic) terms in the expansion of the energy of the crystal with respect to the displacements of particles from their equilibrium positions result in phonon-phonon interactions and, therefore, in the renormalization of the phonon frequencies. At a given quasimomentum $\mathbf{k}$, the renormalized frequencies correspond to the poles of the Fourier transform

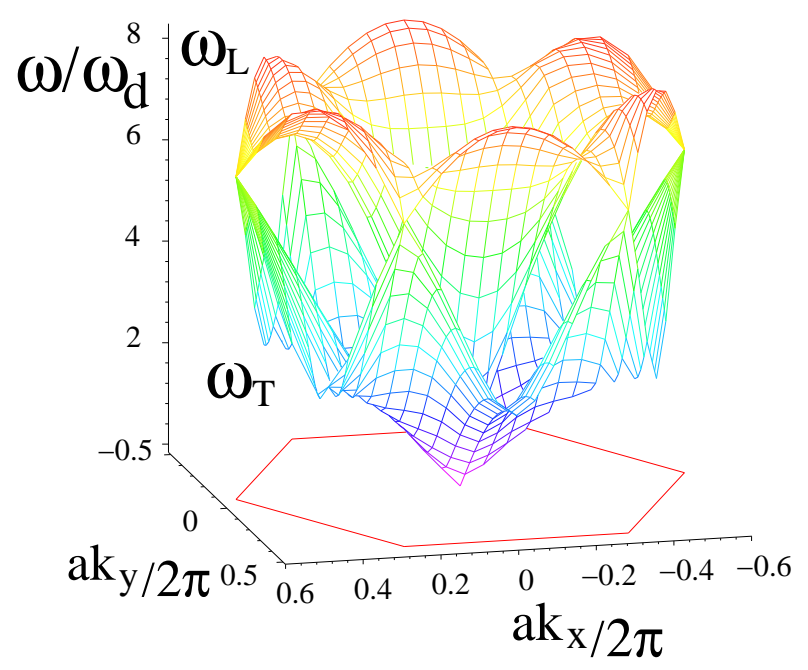

Figure 2: Energy of the transverse $\omega_{T}$ (lower surface) and longitudinal $\omega_{L}$ phonons.

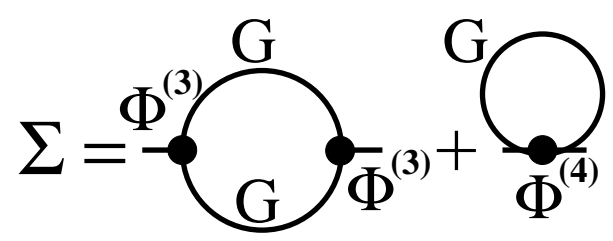

Figure 3: One-loop diagrams for the phonon self-energy.

$G_{\alpha \beta}(\omega, \mathbf{k})$ of the phonon Green function $G_{\alpha \beta}\left(t, \mathbf{l}-\mathbf{l}^{\prime}\right)=$ $i\left[\theta(t)\left\langle u_{\alpha \mathbf{l}}(t) u_{\beta \mathbf{1}^{\prime}}(0)\right\rangle+\theta(-t)\left\langle u_{\beta \mathbf{1}^{\prime}}(0) u_{\alpha \mathbf{l}}(t)\right\rangle\right]$ with $\theta(t)$ being the step function (for more details on the usage of Green functions for phonons in crystals see e.g. 23]). The Green function in the harmonic approximation is $G_{\alpha \beta}^{(0)-1}(\omega, \mathbf{k})=\left\{\sum_{s=T, L} \mathcal{M}_{\alpha \beta}^{(s)}\left[\omega_{s}^{2}(\mathbf{k})-\omega^{2}\right]-\right.$ $\left.i \varepsilon_{\alpha \beta} \omega_{c} \omega\right\} m / \hbar$, where $\mathcal{M}_{\alpha \beta}^{(s)}=e_{\alpha}^{(s)} e_{\beta}^{(s)}$ is the projector to the eigenmode $s$ with the polarization $\mathbf{e}^{(s)}$, and the poles are at $\omega=\omega_{ \pm}(\mathbf{k})$. In the presence of phonon-phonon interactions, the Green function obeys the Dyson equation

$$
G_{\alpha \beta}^{-1}(\omega, \mathbf{k})=G_{\alpha \beta}^{(0)-1}(\omega, \mathbf{k})-\Sigma_{a \beta}(\omega, \mathbf{k}),
$$

where the phonon self-energy function $\Sigma$ incorporates all the effects of phonon-phonon interactions.

We calculate the phonon self-energy in the one-loop approximation that has proven its reliability in the case of an electron Wigner crystal in a strong magnetic field [24, 25, 26]. This corresponds to taking into account only the fourth $\Phi^{(4)} \sim \partial^{4} U_{\mathrm{C}} / \partial R^{4}$ and the square of the third $\Phi^{(3)} \sim \partial^{3} U_{\mathrm{C}} / \partial R^{3}$ order anharmonic terms, see Fig 3 , We then solve the Dyson equation numerically by successive iterations until we either obtain a self-consistent solution or the iteration breaks down due to the appearance of purely imaginary phonon frequencies. The latter indicates the instability of the lattice. The results 
of our calculations can be summarized as follows. At very low filling factors the phonon-phonon interactions do not play any significant role and the system behaves harmonically. However, the effects of anharmonicity become progressively important with increasing the filling factor, and, finally, at some critical filling factor $\nu_{c}$ we find purely imaginary frequencies and, hence, the crystal ceases to exist. We always observe this phonon instability for $k \rightarrow 0$. This indicates the breakdown of the crystalline order and rules out any possible structural phase transition. Therefore, the value $\nu_{c}$ sets the upper bound for the stability of the crystal. The critical filling factor $\nu_{c}$ depends on the confinement in axial direction as well as on the ratio of $\omega_{d}$ and $\omega_{c}$ that measures the strength of the dipole-dipole interaction relative to the Landau level spacing. This dependence for $l=0$ can be approximated as $\nu_{c}^{-1}=4.33 \exp \left(-0.0021 \omega_{c} / \omega_{d}\right)+5.77$. Note that for $\omega_{c} \gg \omega_{d}$ the critical filling factor $\nu_{c}$ becomes insensitive to the strength of the interparticle interaction. In this limit the Wigner crystal is stable for $\nu<\nu_{c}=0.174$, which is in a good agreement with our previous energetic consideration for the tight confinement along the $z$-axis, $l<l_{0}$.

We also calculate the Lindemann parameter $\gamma$ - the ratio of the average displacement of a particle in the lattice from its equilibrium position to the lattice spacing $a, \gamma=\sqrt{\left\langle\mathbf{u}^{2}\right\rangle} / a$. For $\omega_{c} \gg \omega_{d}$ we find $\gamma=0.28$ that is within the range of values of the Lindemann parameter for various $2 \mathrm{D}$ crystals.

As we see, the crucial parameter that controls the ground state of the system and, therefore, drives the quantum melting transition is the filling factor. The way of manipulating the filling factor in experiments with rotating gases depends on an experimental setup. In the case of a critical rotation with an extra (quartic) confinement [27] this can be achieved by changing the number of particles (the size of the system is fixed by an extra confinement). For a purely harmonic confinement and "under critical" rotations [28, 29], the filling factor can be changed by varying the difference $\Omega-\omega_{\perp}$. In this setup, the size of the system and, therefore, the filling factor is determined by a competition between the interparticle interaction $V_{\mathrm{D}}$ and the "tilting" term $\left(\Omega-\omega_{\perp}\right) L_{z}$ (see Eq. 2). In both cases, the appearance of a crystal order could be detected by studying the shot noise correlations using the Hanbury Brown and Twiss effect [30] in a similar way as it was used to observe the Mott insulator-superfluid transition in a lattice Bose gas [31].

In conclusion, we show the existence of the Wigner crystal state for low filling factors in a rapid rotating $2 \mathrm{D}$ dipolar Fermi gas. The critical filling factor, at which the quantum melting of the crystal into a quantum Hall liquid takes place, depends on the confinement in the axial direction and on the strength of the dipole-dipole interaction, but typically one expects a liquid ground state for $\nu>1 / 7$.
We wish to acknowledge N. Barberàn, N.R. Cooper, J.M.F. Gunn, E. Jeckelmann, A.H. MacDonald, K. Osterloh, and R. Wimmer for helpful discussions. This work was supported by the Nederlandse Stichting voor Fundamenteel Onderzoek der Materie (FOM), the Russian Foundation for Fundamental Research, the Deutsche Forschungsgemeinschaft (SPP1116, SFB 407, GK 282), ESF PESC "QUDEDIS", EU IP "SCALA", Spanish MEC (FIS 2005-04627 and Consolider-Ingenio 2010 "QOIT").

[1] M. Greiner et al., Nature 415, 39 (2002); M. Greiner et al., Nature 419, 51 (2002).

[2] I. Bloch and M. Greiner, Adv. At. Molec. Opt. Phys. 52, 1 (2005).

[3] M. Lewenstein et al., Adv. Phys. 56, 243 (2007).

[4] D. C. Tsui, H. L. Stormer, and A.C. Gossard, Phys. Rev. Lett. 48, 1559 (1982).

[5] R. B. Laughlin, Phys. Rev. Lett. 50, 1395 (1983).

[6] E. Wigner, Phys. Rev. 46, 1002 (1934).

[7] N.K. Wilkin, and J.M.F. Gunn, Phys. Rev. Lett. 84, 6 (2000); N.R. Cooper, N.K. Wilkin, and J.M.F. Gunn, Phys. Rev. Lett. 87, 120405 (2001); Tin-Lun Ho, Phys. Rev Lett. 87, 060403 (2001).

[8] M. A. Baranov, K. Osterloh, and M. Lewenstein, Phys. Rev. Lett. 94, 070404 (2005).

[9] N.R. Cooper, E.H. Rezayi, and S.H. Simon, Phys. Rev. Lett. 95, 200402 (2005).

[10] S. Komineas and N.R. Cooper, Phys. Rev. A 75, 023623 (2007).

[11] E.H. Rezayi, N. Read and N.R. Cooper, Phys. Rev. Lett 95, 160404 (2005).

[12] S. Das Sarma and A. Pinczuk (Eds.), Perspectives in Quantum Hall Effects: Novel Quantum Liquids in LowDimensional Semiconductor Structures, (Wiley, New York, 1996).

[13] H.P. Büchler et al., Phys. Rev. Lett 98, 060404 (2007); G.E. Astrakharchik et al., Lett 98, 060405 (2007).

[14] A. Griesmaier, J. Werner, S. Hensler, J. Stuhler, and T. Pfau, Phys. Rev. Lett. 94, 160401 (2005).

[15] T. Lahaye et al., Nature 448, 672 (2007).

[16] Special issue on Ultracold Polar Molecules: Formation and Collisions, Eur. Phys. J. D, 31 (2004).

[17] K. Maki and X. Zotos, Phys. Rev. B 28, 4349 (1983).

[18] K. Osterloh, N. Barberán, and M. Lewenstein, to be published

[19] J.K. Jain, Phys. Rev. Lett 63, 199 (1989).

[20] Pui K. Lam and S.M. Girvin, Phys. Rev. B 30, 473 (1984); Hangmo Yi and H.A. Fertig, Phys. Rev. B 58, 4019 (1988).

[21] H. Fukuyama, Solid State Com. 17, 1323 (1975).

[22] A.V. Chaplik, Sov. Phys.-JETP 35, 395 (1972).

[23] H. Böttger, Principles of the Theory of Lattice Dynamics, Physik Verlag, Weinheim, 1983.

[24] V. M. Bedanov, G. V. Gadiyak, and Yu. E. Lozovik, Physics Letters A 109, 289 (1985).

[25] Y. E. Lozovik, D. R. Musin, and V. I. Yudson, Sov. Phys. Solid State 21, 1132 (1979).

[26] Y. E. Lozovik, V. M. Farztdinov, and A. Abdullaev, J. 
Phys. C: Solid State Phys. 18, L807 (1985).

[27] V. Bretini et al., Phys. Rev. Lett. 92, 050403 (2004).

[28] J. R. Abo-Shaeer et al., Science 292, 476 (2001).

[29] V. Schweikhard et al., Phys. Rev. Lett. 92, 040404 (2004).

[30] R. Hanbury Brown and R. Q. Twiss, Nature 117, 27
(1956).

[31] S. Fölling et al., Nature 434, 481 (2005).

[32] The filling factor $\nu$ denotes the fraction of occupied Landau levels. For a rotating Bose gas it is the number of vortices per atom. 